\title{
The heavy mesons in Nambu-Jona-Lasinio model
}

\author{
Xiao-Yu Guo, Xiao-Lin Chen, and Wei-Zhen Deng* \\ School of physics and State Key Laboratory of Nuclear Physics and Technology, Peking University, Beijing, 100871, China
}

\begin{abstract}
We propose an extended Nambu-Jona-Lasinio model to include the heavy mesons with the heavy quark symmetry. The quark current-current interaction is generalized to include the heavy quark currents. In order to comply with the heavy quark spin symmetry at the heavy quark limit, the quark mass dependence of the interaction strength is introduced. The light and heavy pseudo-scalar and vector meson, their masses and the weak decay constants, are calculated in the unified frame.
\end{abstract}

PACS numbers: 12.39.Fe, 12.39.Hg, 14.40.-n

Keywords: NJL model, heavy meson, heavy quark limit

\section{INTRODUCTION}

In recent years, some exotic hadron states have been observed in experiments. Many of them cannot be explained easily as the conventional quarkonia. A possible interpretation is the hypothesis of molecular state [1 3]. Many of the studies on the exotic states were based on the heavy quark effective theory (HQET). Recently the chiral quark model have been used in solving the molecular state [4, 5]. Currently, the most difficulty to identify a molecular state is the uncertainty of the parameters about the interaction strengths and the form factors.

On principle these parameters can be calculated from QCD on the quark level. However, in the low-energy region where the QCD perturbation method fails we have to rely on effective theories. Among them, the Nambu-JonaLasinio (NJL) model [6, 7] was widely used to investigate many low-energy hadron problems related to the QCD symmetries in a simple way [8].

By means of Dyson-Schwinger equation (DSE), the dynamic quark mass is generated from the spontaneous chiral symmetry breaking. After solving the Bethe-Salpeter equation (BSE), pseudo-scalar mesons are obtained as the Goldstone bosons [6, 7]. Other mesons such as vector mesons and axial-vector mesons were included by introducing more chiral invariant interactions [11 13]. Also the model was extended to comprise the strange flavor [14, 15]. A bosonization technique was also developed [16] and many works were done along this approach [17 20].

Because of the QCD color coulomb interaction, a heavy quark spin symmetry is reached in the heavy quark limit that the dependency of hadronic matrix elements on the orientation of the heavy quark spin vanishes [21]. From the heavy quark symmetry the HQET formalism was developed (for a review, see Refs. 22, 23]).

Some efforts were made on studying the heavy mesons within the NJL model [24, 25]. The bosonization technique was used in these studies to obtained the meson Lagrangian in HQET. Using the heavy quark propagators in the heavy quark limit, the DSE+BSE approach was also been used to calculate heavy meson observables [26, 27.

In the NJL model study, the color-octet vector current interaction $\left(\bar{\psi} \lambda_{C}^{a} \gamma_{\mu} \psi\right)\left(\bar{\psi} \lambda_{C}^{a} \gamma^{\mu} \psi\right)$ was widely adopted since it is closely related to the QCD interaction. In many DSE+BSE calculations such as in Ref. 28], the interaction between two quarks was assumed to be intermediated by the gluon with a complicated effective propagator. So the color-octet vector current of the quark should be dominant. The DSE+BSE calculation using the gluon propagator was also performed in the heavy meson case [29]. If we naively treat the gluon propagator as a constant in the coordinate space, we would obtain an NJL model with the color-octet vector current interaction. In the heavy quark limit where the heavy quark mass $m_{Q}$ tends to infinity, we will show that the heavy quark spin symmetry is valid only for the color-octet vector current interaction.

Other contact interactions such as the color-octet axial-vector current interaction $\left(\bar{\psi} \lambda_{C}^{a} \gamma_{\mu} \gamma_{5} \psi\right)\left(\bar{\psi} \lambda_{C}^{a} \gamma^{\mu} \gamma_{5} \psi\right)$ are needed to give a more comprehensive description of the light flavor mesons such as the $\rho$ meson [11 15]. We will show that the heavy quark spin symmetry would not be reached if these interactions exist in the heavy quark limit. To maintain this symmetry, these interactions should be considered as higher order terms and should be $1 / m_{Q}$ suppressed. This is critical to extend the NJL model to include heavy quark flavors.

We will extend the NJL model to include the heavy quark flavors. The typical approach of DSE+BSE will be used to obtain properties of heavy mesons. In this way, we can calculate the mass splitting between the pseudo-scalar mesons $D$ (or $B$ ) and the vector mesons $D^{*}$ (or $B^{*}$ ) which is the effect of finite heavy quark mass according to the

*Electronic address: dwz@pku.edu.cn 
heavy quark expansion. Due to the fact that the heavy quark masses are far beyond the NJL cutoff scale, the usual 4-dimensional cutoff is not appropriate here. We will use the 3-dimensional cutoff following Refs. [6, 7, 11].

In the next section, we will generalize the NJL interaction to include the heavy quark flavor and derive the mass dependence of the coupling strength parameters according to the heavy quark spin symmetry. In Section III, we will give a brief account of the DSE+BSE formalism to treat the quark and meson states. In Section IV] we will take the heavy quark limit and demonstrate the heavy quark spin symmetry. In Section $\mathrm{V}$, numerical calculation will be performed and the result will be compared to the empirical data. Finally we will give a brief summary.

\section{NJL INTERACTION WITH HEAVY QUARK SYMMETRY}

In many NJL studies, when dealing with the three light flavors $q=u, d, s$, the interaction was taken to be the color current interaction

$$
\mathcal{L}_{4}=G_{V}\left(\bar{q} \lambda_{C}^{a} \gamma_{\mu} q\right)^{2}+G_{A}\left(\bar{q} \lambda_{C}^{a} \gamma_{\mu} \gamma_{5} q\right)^{2} .
$$

The interaction maintains the $U_{f}(3) \otimes U_{f}(3) \otimes S U_{C}(3)$ symmetry. Here we will not consider the 6 -quark interaction which was used to deal the $U_{A}(1)$ anomaly, since we will not concern the anomaly here and the contribution of the anomaly term is small [15]. After a Fierz transformation, we can get a Fierz invariant interaction

$$
\mathcal{L}_{4}^{F}=\frac{4}{9} G_{1} \sum_{i=0}^{8}\left[\left(\bar{q} \lambda_{f}^{i} q\right)^{2}+\left(\bar{q} i \gamma_{5} \lambda_{f}^{i} q\right)^{2}\right]-\frac{2}{9} G_{2} \sum_{i=0}^{8}\left[\left(\bar{q} \lambda_{f}^{i} \gamma_{\mu} q\right)^{2}+\left(\bar{q} \lambda_{f}^{i} \gamma_{\mu} \gamma_{5} q\right)^{2}\right]+\text { color-octet terms }
$$

where

$$
G_{1}=G_{V}-G_{A}, \quad G_{2}=G_{V}+G_{A}
$$

Here the $\lambda_{i}$ 's are the flavor Gell-Mann matrices with $\lambda_{0} \equiv \sqrt{\frac{2}{3}} \mathbb{1}$. The color-octet terms do not contribute to the DSE+BSE calculation of the meson.

In Ref. [24], where the heavy flavors $Q=c, b$ were considered, only the color-octet vector interaction $\left(\bar{q} \lambda_{C}^{a} \gamma_{\mu} q\right)\left(\bar{Q} \lambda_{C}^{a} \gamma^{\mu} Q\right)$ was considered. In Section IV] we will show that only this term in Eq. (1) respects the heavy quark spin symmetry in the heavy quark limit.

The color-octet vector interaction is however not enough to describe the light flavor mesons such as the vector $\rho$ meson. We will also show that the heavy quark spin symmetry would not be reached if other than the vector interaction exists in the heavy quark limit. To consistently describe the light sector and the heavy sector of the meson system, we assume that the NJL interaction is originated in the color-octet vector current. Other currents appear as higher order correction in some series expansion and thus should be suppressed by the $1 / m_{q}$ factor if the expansion is taken with respect to the constituent quark mass $m_{q}$. According to this thought, we modify the NJL interaction Eq. (11) to

$$
\mathcal{L}_{4}=G_{V}\left(\bar{q} \lambda_{C}^{a} \gamma_{\mu} q\right)\left(\bar{q}^{\prime} \lambda_{C}^{a} \gamma_{\mu} q^{\prime}\right)^{2}+\frac{h_{1}}{m_{q} m_{q^{\prime}}}\left(\bar{q} \lambda_{C}^{a} \gamma_{\mu} q\right)\left(\bar{q}^{\prime} \lambda_{C}^{a} \gamma_{\mu} q^{\prime}\right)+\frac{h_{2}}{m_{q} m_{q^{\prime}}}\left(\bar{q} \lambda_{C}^{a} \gamma_{\mu} \gamma_{5} q\right)\left(\bar{q}^{\prime} \lambda_{C}^{a} \gamma_{\mu} \gamma_{5} q^{\prime}\right) .
$$

Here we can take the light and heavy quarks into a unified frame $q, q^{\prime}=u, d, s, c, b$. $h_{1}$ and $h_{2}$ are dimensionless parameters. $m_{q}$ and $m_{q^{\prime}}$ are the constituent masses of the quarks involved in the interaction.

The Fierz invariant interaction Eq. (2) has the interaction strengths

$$
G_{1}=G_{V}+\frac{h_{1}-h_{2}}{m_{q} m_{q^{\prime}}}, \quad G_{2}=G_{V}+\frac{h_{1}+h_{2}}{m_{q} m_{q^{\prime}}} .
$$

We notice that $G_{1}$ is tightly related to the quark constituent mass which is the dynamical one generated from the gap equation (see Eqs. (9) and (10) in Section (III). If $G_{1}$ depends on the constituent quark mass, the gap equation will change radically. As shown in FIG. 1, when $h_{1}=h_{2}$, the case of usual NJL model where $G_{1}=G_{V}$ is independent on the constituent quark mass, the gap equation has the two solutions corresponding to two chiral phases which is believed to exist in the QCD chiral limit: a Wigner solution at $m_{q}=0$ and a chiral symmetry breaking solution at $m_{q} \neq 0$ when the coupling is large enough.

However, when $h_{1} \neq h_{2}$, the gap equation reveals a singularity at $m_{q}=0$. Hence, there is no chiral phase (Wigner solution). So we must set $h_{1}=h_{2}=h$ and the NJL interaction turns to be

$$
\mathcal{L}_{4}=G_{V}\left(\bar{\psi} \lambda_{C}^{a} \gamma_{\mu} \psi\right)^{2}+\frac{h}{m_{q} m_{q^{\prime}}}\left[\left(\bar{\psi} \lambda_{C}^{a} \gamma_{\mu} \psi\right)^{2}+\left(\bar{\psi} \lambda_{C}^{a} \gamma_{\mu} \gamma_{5} \psi\right)^{2}\right]
$$



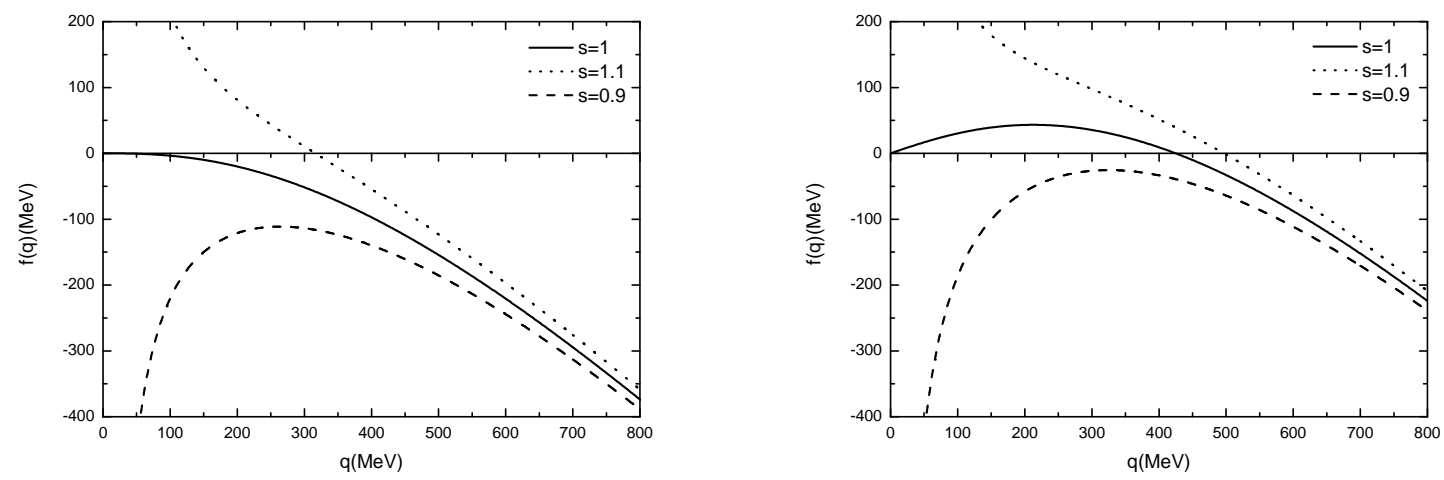

FIG. 1: The gap equation where the zero points are the solution of quark mass. The cutoff is taken at $\Lambda=750 \mathrm{MeV}$. $h_{2}=0.65, h_{1}=s h_{2}$. The dimensionless parameter $g_{V}$ is defined as $g_{V}=G_{V} \Lambda^{2}$. We show two typical situations in the figure: $g_{V}=g c=9 \pi^{2} / 16 N_{C}$ where the strength is critically not enough to break the chiral symmetry; and $g_{V}=2.5$ with the chiral symmetry breaking solution.

After the Fierz transformation, we obtain the relevant Fierz invariant interaction: for the light sector

$$
\mathcal{L}_{4}^{F}=\frac{4}{9} G_{V}\left[\left(\bar{q} \lambda_{f}^{i} q\right)^{2}+\left(\bar{q} i \gamma_{5} \lambda_{f}^{i} q\right)^{2}\right]-\frac{2}{9}\left(G_{0}+\frac{2 h}{m_{q} m_{q^{\prime}}}\right)\left[\left(\bar{q} \lambda_{f}^{i} \gamma_{\mu} q\right)^{2}+\left(\bar{q} \lambda_{f}^{i} \gamma_{\mu} \gamma_{5} q\right)^{2}\right]
$$

and for the heavy sector

$$
\mathcal{L}_{4}^{F^{\prime}}=\frac{8}{9} G_{V}\left[(\bar{Q} q)(\bar{q} Q)+\left(\bar{Q} i \gamma_{5} q\right)\left(\bar{q} i \gamma_{5} Q\right)\right]-\frac{4}{9}\left(G_{0}+\frac{2 h}{m_{q} m_{Q}}\right)\left[\left(\bar{Q} \gamma_{\mu} q\right)\left(\bar{q} \gamma^{\mu} Q\right)+\left(\bar{Q} \gamma_{\mu} \gamma_{5} q\right)\left(\bar{q} \gamma_{\mu} \gamma_{5} Q\right)\right] .
$$

Because the difference between the constituent mass and current mass of heavy quark should be small, we will ignore the dynamical effect upon the heavy quark mass in the calculation of heavy mesons.

\section{BETHE-SALPETER EQUATION AND MESONS}

Now we will give a brief account of the method of DSE and BSE used in our calculation of meson states. Throughout this section we will use Eq. (2) as a general form of the NJL interaction.

The Dyson-Schwinger Equation (DSE) is used to obtain the dynamical quark mass $m_{q}$. The self-consistent gap equation derived from DSE reads

$$
m_{q}=m_{q}^{0}+\Sigma_{q},
$$

where $m_{q}^{0}$ is the current quark mass and $\Sigma_{q}$ is the quark self energy

$$
-i \Sigma_{q}=i \frac{32}{9} G_{1} \operatorname{Tr} \int \frac{\mathrm{d}^{4} p}{(2 \pi)^{4}} S_{q}(p)=-\frac{16 G_{1}}{9} m_{q} I_{1}\left(m_{q}\right),
$$

where $S_{q}(p)$ is the quark propagator. The expression of the integral $I_{1}\left(m_{q}\right)$ is given in the Appendix.

We use Bethe-Salpeter equation (BSE) to obtain the meson mass and amplitude. The total quark anti-quark scattering amplitude is obtained from the ladder approximation. We decompose the amplitude into different Lorentz structures [15]. The relevant amplitudes are

$$
\begin{aligned}
\mathcal{T}_{\mathrm{ps}} & =T_{P P}\left(i \gamma_{5} \lambda_{i} \otimes i \gamma_{5} \lambda_{j}\right)+T_{A P}\left(-i \not \gamma_{5} \lambda_{i} \otimes i \gamma_{5} \lambda_{j}\right)+T_{P A}\left(i \gamma_{5} \lambda_{i} \otimes i \not \gamma_{5} \lambda_{j}\right)+T_{A A}^{P}\left(-i \not \gamma_{5} \gamma_{i} \otimes i \not \gamma_{5} \lambda_{j}\right), \\
\mathcal{T}_{\mathrm{v}} & =T_{V V}\left(\eta^{\mu \nu} \gamma_{\mu} \lambda_{i} \otimes \gamma_{\nu} \lambda_{j}\right),
\end{aligned}
$$

where $\hat{q}^{\mu}=q^{\mu} / \sqrt{q^{2}}, \eta_{\mu \nu}=g_{\mu \nu}-\hat{q}^{\mu} \hat{q}^{\nu}$. In the ladder approximation, we need only calculate the loop integral

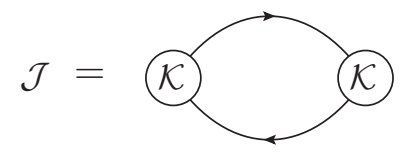


which can also be decomposed to

$$
\begin{aligned}
\mathcal{J}_{\mathrm{ps}}^{i j}= & J_{P P}\left(i \gamma_{5} \lambda_{i} \otimes i \gamma_{5} \lambda_{j}\right)+J_{A P}\left(-i \not \gamma_{5} \lambda_{i} \otimes i \gamma_{5} \lambda_{j}\right) \\
& +J_{P A}\left(i \gamma_{5} \lambda_{i} \otimes i \not \gamma_{5} \lambda_{j}\right)+J_{A A}^{L}\left(-i \not \gamma_{5} \lambda_{i} \otimes i \not q \gamma_{5} \lambda_{j}\right), \\
\mathcal{J}_{\mathrm{v}}^{i j}= & \eta^{\mu \nu} J_{V V}^{T}\left(\gamma_{\mu} \lambda_{i} \otimes \gamma_{\nu} \lambda_{j}\right) .
\end{aligned}
$$

Then we have

$$
T=\frac{1}{1-J K}
$$

where

$$
\begin{array}{ll}
K_{\mathrm{P}}=\frac{16 G_{1}}{9}\left(i \gamma_{5} \lambda_{i} \otimes i \gamma_{5} \lambda_{j}\right), & K_{\mathrm{S}}=\frac{16 G_{1}}{9}\left(\lambda_{i} \otimes \lambda_{j}\right) \\
K_{\mathrm{A}}=-\frac{8 G_{2}}{9}\left(\gamma_{\mu} \gamma_{5} \lambda_{i} \otimes \gamma_{\nu} \gamma_{5} \lambda_{j}\right), & K_{\mathrm{V}}=-\frac{8 G_{2}}{9}\left(\gamma_{\mu} \lambda_{i} \otimes \gamma_{\nu} \lambda_{j}\right)
\end{array}
$$

The integrals $J_{A B}$ are defined in ref. [15] and formulae with 3-dimensional cut-off are collected in the Appendix.

The meson mass $m_{M}$ is determined by the pole of the amplitude,

$$
\left.\operatorname{Det}(1-J K)\right|_{q^{2}=m_{M}^{2}}=0 \text {. }
$$

To calculate the weak decay constant of a pseudo-scalar meson, the quark-meson vertex is obtained by expanding the scattering amplitude near the meson pole. For a pseudo-scalar meson $P$, which could be $\pi, K, D$, or $B$, the $q q P$ vertex reads

$$
V_{P}^{i}(p)=i \gamma_{5} \lambda^{i}\left[g_{P}\left(p^{2}\right)-\frac{\not p}{m_{q}+m_{q^{\prime}}} \tilde{g}_{P}\left(p^{2}\right)\right]
$$

where

$$
\begin{aligned}
& g_{P}^{2}=\left(\frac{\mathrm{d} D}{\mathrm{~d} q^{2}}\right)_{q^{2}=m_{P}^{2}}^{-1} K_{P}\left(1-J_{A A} K_{A}\right), \\
& \tilde{g}_{P}=\frac{m_{q}+m_{q^{\prime}}}{m_{P}} \frac{K_{A} J_{P A}}{1-J_{A A} K_{A}} g_{P},
\end{aligned}
$$

where $D=\operatorname{Det}(1-J K)$. The pion decay constant is given by

$$
\left\langle 0\left|\bar{q}(0) \gamma^{\mu} \gamma_{5} \frac{\lambda_{i}}{2} q(0)\right| \pi_{j}(p)\right\rangle=i f_{\pi} p^{\mu} \delta_{i j}
$$

Similar result holds for the kaon decay constant. In the heavy quark case the decay constant is given by

$$
\left\langle 0\left|\bar{q}(0) \gamma^{\mu} \gamma_{5} Q(0)\right| H(p)\right\rangle=i F_{H} p^{\mu},
$$

where $H$ could be $D$ or $B$.

\section{HEAVY QUARK LIMIT}

In this section, we will discuss the heavy quark limit. After the Fierz transformation, the relevant interaction between a light quark $q$ and a heavy quark $Q$ in a heavy meson is written in the form

$$
\mathcal{L}_{4}^{F^{\prime}}=\frac{8}{9} G_{1}\left[(\bar{Q} q)(\bar{q} Q)+\left(\bar{Q} i \gamma_{5} q\right)\left(\bar{q} i \gamma_{5} Q\right)\right]-\frac{4}{9} G_{2}\left[\left(\bar{Q} \gamma_{\mu} q\right)\left(\bar{q} \gamma^{\mu} Q\right)+\left(\bar{Q} \gamma_{\mu} \gamma_{5} q\right)\left(\bar{q} \gamma_{\mu} \gamma_{5} Q\right)\right]
$$

Consider the heavy meson at rest, $q=m_{H} v, v=(1,0,0,0)$. In the heavy quark limit one assumes that the mass difference between $m_{H}$ of the heavy meson and $m_{Q}$ of the heavy quark is a small quantity $l_{0}$,

$$
m_{H}=m_{Q}+l_{0}
$$


The heavy quark momentum $p$ is expanded around the heavy meson momentum $q$ as $p=q+k$, where $k$ is assumed to be far smaller than $m_{Q}$. Then the propagator of the heavy quark reduces to

$$
\frac{1}{(\not k+\not 1)-m_{Q}} \approx \frac{\psi+1}{2\left(k \cdot v+l_{0}\right)} .
$$

The expression on the right hand side is independent of $m_{Q}$. The BSE loop integrals reduce to

$$
\begin{aligned}
& J_{P P}=2 i N_{C} \operatorname{tr} \int \frac{\mathrm{d}^{4} k}{(2 \pi)^{4}} i \gamma_{5} \frac{1}{\not k-m_{q}+i \epsilon} i \gamma_{5} \frac{\psi+1}{2\left(k \cdot v+l_{0}+i \epsilon\right)}, \\
& J_{P A}=2 i N_{C} \operatorname{tr} v^{\mu} \int \frac{\mathrm{d}^{4} k}{(2 \pi)^{4}} i \gamma_{5} \frac{1}{\not k-m_{q}+i \epsilon}\left(-i \gamma_{\mu} \gamma_{5}\right) \frac{\psi+1}{2\left(k \cdot v+l_{0}+i \epsilon\right)}, \\
& J_{S S}=2 i N_{C} \operatorname{tr} \int \frac{\mathrm{d}^{4} k}{(2 \pi)^{4}} \frac{1}{\not k-m_{q}+i \epsilon} i \gamma_{5} \frac{\psi+1}{2\left(k \cdot v+l_{0}+i \epsilon\right)}, \\
& J_{S V}=2 i N_{C} \operatorname{tr} v^{\mu} \int \frac{\mathrm{d}^{4} k}{(2 \pi)^{4}} \frac{1}{\not k-m_{q}+i \epsilon} \gamma_{\mu} \frac{\psi+1}{2\left(k \cdot v+l_{0}+i \epsilon\right)}, \\
& J_{V V}^{\mu \nu}=2 i N_{C} \operatorname{tr} \int \frac{\mathrm{d}^{4} k}{(2 \pi)^{4}} \gamma^{\mu} \frac{1}{\not k-m_{q}} \gamma^{\nu} \frac{\psi+1}{2\left(k \cdot v+l_{0}\right)}, \\
& J_{A A}^{\mu \nu}=2 i N_{C} \operatorname{tr} \int \frac{\mathrm{d}^{4} k}{(2 \pi)^{4}} \gamma^{\mu} \gamma_{5} \frac{1}{\not k-m_{q}} \gamma^{\nu} \gamma_{5} \frac{\psi+1}{2\left(k \cdot v+l_{0}\right)} .
\end{aligned}
$$

We find

$$
\begin{aligned}
J_{P P}=J_{P A} & =4 i N_{C} \int \frac{\mathrm{d}^{4} k}{(2 \pi)^{4}} \frac{k \cdot v-m_{q}}{\left(k^{2}-m_{q}^{2}+i \epsilon\right)\left(v \cdot k+l_{0}+i \epsilon\right)}, \\
J_{S S}=J_{S V} & =4 i N_{C} \int \frac{\mathrm{d}^{4} k}{(2 \pi)^{4}} \frac{k \cdot v+m_{q}}{\left(k^{2}-m_{q}^{2}+i \epsilon\right)\left(v \cdot k+l_{0}+i \epsilon\right)}, \\
J_{V V}^{\mu \nu} & =4 i N_{C} \int \frac{\mathrm{d}^{4} k}{(2 \pi)^{4}} \frac{k^{\mu} v^{\nu}+v^{\mu} k^{\nu}-g^{\mu \nu} k \cdot v+g^{\mu \nu} m_{q}}{\left(k^{2}-m_{q}^{2}\right)\left(v \cdot k+l_{0}\right)}, \\
J_{A A}^{\mu \nu} & =4 i N_{C} \int \frac{\mathrm{d}^{4} k}{(2 \pi)^{4}} \frac{k^{\mu} v^{\nu}+v^{\mu} k^{\nu}-g^{\mu \nu} k \cdot v-g^{\mu \nu} m_{q}}{\left(k^{2}-m_{q}^{2}\right)\left(v \cdot k+l_{0}\right)} .
\end{aligned}
$$

After further decompositions $J_{V V}^{\mu \nu}=J_{V V}^{T}\left(g^{\mu \nu}-v^{\mu} v^{\nu}\right)+J_{V V}^{L} v^{\mu} v^{\nu}$ and $J_{A A}^{\mu \nu}=J_{A A}^{T}\left(g^{q \mu \nu}-v^{\mu} v^{\nu}\right)+J_{A A}^{L} v^{\mu} v^{\nu}$, we have

$$
\begin{aligned}
& J_{V V}^{L}=4 i N_{C} \int \frac{\mathrm{d}^{4} k}{(2 \pi)^{4}} \frac{k \cdot v+m_{q}}{\left(k^{2}-m_{q}^{2}\right)\left(v \cdot k+l_{0}\right)}, \\
& J_{V V}^{T}=4 i N_{C} \int \frac{\mathrm{d}^{4} k}{(2 \pi)^{4}} \frac{-k \cdot v+m_{q}}{\left(k^{2}-m_{q}^{2}\right)\left(v \cdot k+l_{0}\right)}, \\
& J_{A A}^{L}=4 i N_{C} \int \frac{\mathrm{d}^{4} k}{(2 \pi)^{4}} \frac{k \cdot v-m_{q}}{\left(k^{2}-m_{q}^{2}+i \epsilon\right)\left(v \cdot k+l_{0}+i \epsilon\right)}, \\
& J_{A A}^{T}=4 i N_{C} \int \frac{\mathrm{d}^{4} k}{(2 \pi)^{4}} \frac{-k \cdot v-m_{q}}{\left(k^{2}-m_{q}^{2}+i \epsilon\right)\left(v \cdot k+l_{0}+i \epsilon\right)} .
\end{aligned}
$$

Thus, in the heavy quark limit

$$
\begin{gathered}
J_{P P}\left(l_{0}\right)=J_{P A}\left(l_{0}\right)=J_{A A}^{L}\left(l_{0}\right)=-J_{V V}^{T}\left(l_{0}\right), \\
J_{S S}\left(l_{0}\right)=J_{S V}\left(l_{0}\right)=J_{V V}^{L}\left(l_{0}\right)=-J_{A A}^{T}\left(l_{0}\right) .
\end{gathered}
$$

For a pseudo-scalar meson, the mass equation Eq. (17) turns to be

$$
\left(1-J_{P P}\left(q^{2}\right) K_{P}\right)\left(1-J_{A A}^{L}\left(q^{2}\right) K_{A}\right)-J_{P A}^{2}\left(q^{2}\right) K_{P} K_{A}=0,
$$

which reduces to

$$
1-\left(K_{P}+K_{A}\right) J_{P P}\left(l_{0}\right)=0
$$


TABLE I: Numerical results of the meson masses and decay constants. The cal. I column: results with the NJL interaction Eq. (6). The cal. II column: results with the interaction Eq. (10) for the light meson sector and the interaction Eq. (23) for the heavy meson sector. The experimental data are taken from Ref. [30] except for $F_{B}$ and $F_{B}^{*}$ which are taken from the lattice calculation in Ref. [31] (see also [32, 33]).

\begin{tabular}{cccc}
\hline \hline & cal. I & cal. II & exp. \\
\hline$m_{u}(\mathrm{MeV})$ & 392 & 389 & 540 \\
$m_{s}(\mathrm{MeV})$ & 542 & 137 & $135 / 140$ \\
$m_{\pi}(\mathrm{MeV})$ & 139 & 496 & $494 / 498$ \\
$m_{K}(\mathrm{MeV})$ & 496 & 86.5 & 93.3 \\
$f_{\pi}(\mathrm{MeV})$ & 91.5 & 88.6 & 114 \\
$f_{K}(\mathrm{MeV})$ & 97.9 & 775 & 775 \\
$m_{\rho}(\mathrm{MeV})$ & 771 & 905 & 892 \\
$m_{K^{*}}(\mathrm{MeV})$ & 918 & 1.86 & $1.86 / 1.87$ \\
$m_{D}(\mathrm{GeV})$ & 1.87 & 1.95 & 1.97 \\
$m_{D_{s}}(\mathrm{GeV})$ & 1.95 & 2.07 & 2.01 \\
$m_{D^{*}}(\mathrm{GeV})$ & 1.99 & 2.20 & 2.11 \\
$m_{D_{s}^{*}}(\mathrm{GeV})$ & 2.12 & 5.28 & 5.28 \\
$m_{B}(\mathrm{GeV})$ & 5.28 & 5.37 & 5.37 \\
$m_{B s}(\mathrm{GeV})$ & 5.37 & 5.36 & 5.33 \\
$m_{B^{*}}(\mathrm{GeV})$ & 5.31 & 5.47 & 5.42 \\
$m_{B_{s}^{*}}(\mathrm{GeV})$ & 5.42 & 123 & 207 \\
$F_{D}(\mathrm{MeV})$ & 139 & 129 & 258 \\
$F_{D s}(\mathrm{MeV})$ & 147 & 87.0 & $190($ lattice) \\
$F_{B}(\mathrm{MeV})$ & 96.7 & 91.8 & $231($ lattice) \\
$F_{B s}(\mathrm{MeV})$ & 107 & & \\
\hline \hline
\end{tabular}

in the heavy quark limit. The mass equation of the vector partner is $1-J_{V V}^{T}\left(q^{2}\right) K_{V}=0$, which leads to

$$
1+K_{V} J_{P P}\left(l_{0}\right)=0
$$

in the heavy quark limit. If $G_{2}=G_{1}$, the mass equations of the pseudo-scalar meson and the vector meson are identical, and the heavy quark spin symmetry is obtained. Otherwise, if $G_{2} \neq G_{1}$, the mass of the pseudo-scalar meson differs from the mass of the vector meson. Similarly, the masses of a scalar meson and its axial-vector partner will be degenerate in the heavy quark limit if and only if $G_{2}=G_{1}$.

\section{NUMERICAL RESULTS}

In the NJL interaction Eq. (6), the input parameters are the current masses for light quarks and constituent masses for heavy quarks, the coupling constants and the 3-dimensional cutoff. We used experimental data of light mesons of $m_{\pi}, m_{K}, m_{\rho}, f_{\pi}$ to determine parameters $m_{u / d}^{0}, m_{s}^{0}, G_{V}, h$ and $\Lambda$. Then the experimental masses of $m_{D}$ and $m_{B}$ is used to determine $m_{c}$ and $m_{b}$. The parameters are

$$
\begin{array}{rlrl}
m_{u / d}^{0} & =2.79 \mathrm{MeV}, & m_{s}^{0} & =72.0 \mathrm{MeV} \\
m_{c} & =1.63 \mathrm{GeV}, \quad m_{b}=4.94 \mathrm{GeV}, \\
\Lambda & =0.8 \mathrm{GeV}, \quad g_{V}=G_{V} \Lambda^{2}=2.41, \quad h=0.65 .
\end{array}
$$

The resulted masses and weak decay constants are show in the cal. I column in TABLE I We find that the meson mass spectra, both the light sector and the heavy sector, are well fitted to the experimental data. One major difficulty is that the calculated decay constant decreases with increasing meson mass while the experimental one increases with mass. As already shown in Ref. [15], the theoretical result of $f_{K}$ is smaller than the empirical data. In the case of heavy mesons, the theoretical results are smaller than the empirical ones by almost a factor 2 . We notice that the decay constant increases with the momentum cutoff parameter $\Lambda$. A possible explanation is that the momentum 

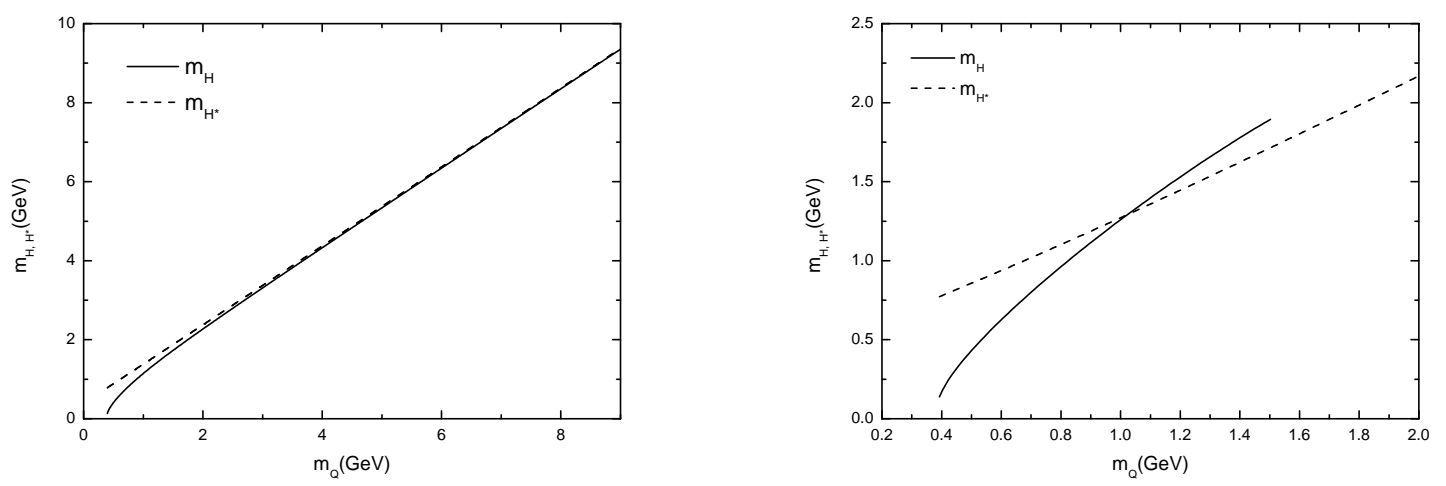

FIG. 2: The dependence of heavy-light meson masses on $m_{Q}$. On the left side, with the interaction Eq. (6). On the right side, with the interaction Eq. (23). The light quark is set to $u$.

cutoff in heavy sector is larger than in light sector which reflects the fact that the size of a heavy meson is relatively small.

The dependence of heavy meson masses on heavy quark mass are plotted in FIG. 2, We use $H$ to represent the heavy pseudo-scalar meson and $H^{*}$ the heavy vector meson. One can see that when the quark mass tends to infinity, the mass splitting between $H$ and $H^{*}$ meson vanishes. On the other hand if we use the interaction in Eq. (23) and keep the parameter $G_{2}$ unchanged vs the quark masses, i.e.

$$
G_{2}=G_{V}+\frac{2 h}{m_{u}^{2}}=5.41 / \Lambda^{2}
$$

we observe a mass crossing of the $H$ meson with $H^{*}$ meson mass as the heavy quark mass increase. Beyond the crossing point, the mass relation is reverted with the $H$ meson above the $H^{*}$. the mass curve of $H$ will further reach the mass threshold and no $H$ bound state exists beyond. So, a naive generalization of the NJL interaction from light quark sector to the heavy is inappropriate.

As a comparison, we also checked with the interaction of a mass independent vector interaction in heavy meson sector, i.e. Eq. (23) with $G_{1}=G_{2}=g_{3} \Lambda^{-2}$. The interaction in light meson sector is Eq. (11) with a different set of couplings $G_{1}=g_{1} \Lambda^{-2}$ and $G_{2}=g_{2} \Lambda^{-2}$. The parameters are

$$
\begin{aligned}
& m_{u / d}^{0}=3.36 \mathrm{MeV}, \quad m_{s}^{0}=81.7 \mathrm{MeV}, \\
& m_{c}=1.68 \mathrm{GeV}, \quad m_{b}=5.00 \mathrm{GeV}, \quad \Lambda=0.7 \mathrm{GeV}, \\
& g_{1}=2.52, \quad g_{2}=5.82 \quad g_{3}=2.53 .
\end{aligned}
$$

The result is shown in the cal. II column in Tab. I) We notice that the mass splitting between the heavy pseudo-scalar meson $D$ (or $B$ ) and its vector partner $D^{*}$ (or $B^{*}$ ) differs from the empirical data by roughly a factor 1.5. For the $D$ and $D^{*}$, it is $210 \mathrm{MeV}$ comparing to the empirical data $150 \mathrm{MeV}$, and for the $B$ and $B^{*}$, it is $80 \mathrm{MeV}$ comparing to $50 \mathrm{MeV}$. To reduce the mass splitting, one may decrease the coupling $g_{V}$. But $g_{V}$ can not be too small, otherwise the interaction will not be strong enough to bound the $D^{*}$ meson.

\section{CONCLUSION}

In this work, we have studied light and heavy mesons in a unified frame with the NJL model. We have followed a traditional approach of solving the DSE and BSE. We have used a 3 dimensional cutoff to adequately regularize the integrals when heavy quarks are involved.

We have investigated the heavy quark spin symmetry in the heavy quark limit. We find that, in the heavy quark limit, the pseudo-scalar meson and its vector partner will have an identical mass equation only if the NJL interaction is a color-octet vector interaction which can be recognized as a approximation of a single-gluon exchange interaction.

Then we propose an extension to the NJL interaction as in Eq. (23) which introduces the $1 / m_{q}$ correction to the quark current. The mass dependence suppresses the axial-vector current interaction to guarantee that the heavy quark spin symmetry still holds in the heavy quark limit. 
We have performed numerical calculations to the light and heavy pseudo-scalar and vector meson, both for their masses and the weak decay constants. The mass spectra fit the experimental data quite well. But the weak decay constants always show a large discrepancy to the experiments. A possible explanation is that the momentum cutoff in heavy sector is larger than in light sector, which reflects the fact that the size of a heavy meson is relatively small. The issue can be studied using some more realistic interaction other than the contact one.

\section{Appendix A: The Current Condensates in 3D Cutoff}

In BSE, we need to calculate the loop integral

$$
J\left(\Gamma, \Gamma^{\prime}, m, m^{\prime}\right)=2 i N_{C} \operatorname{tr} \int \frac{\mathrm{d}^{4} p}{(2 \pi)^{4}}\left[\Gamma \frac{1}{\left(\not p+\frac{1}{2} \not\right)-m+i \epsilon} \Gamma^{\prime} \frac{1}{\left(\not p-\frac{1}{2} \not\right)-m^{\prime}+i \epsilon}\right],
$$

where $\Gamma$ and $\Gamma^{\prime}$ are the interaction vertices. For pseudo-scalar mesons, we have [15]

$$
\begin{aligned}
J_{P P} & =\frac{1}{2}\left[I_{1}(m)+I_{1}\left(m^{\prime}\right)\right]+\left[\left(m-m^{\prime}\right)^{2}-q^{2}\right] I_{2}\left(m, m^{\prime}, q^{2}\right), \\
J_{P A, \mu} & =q_{\mu}\left(m+m^{\prime}\right)\left[1-\frac{\left(m-m^{\prime}\right)^{2}}{q^{2}}\right] I_{2}\left(q^{2}, m, m^{\prime}\right)+q_{\mu} \frac{m-m^{\prime}}{2 q^{2}}\left(I_{1}(m)-I_{1}\left(m^{\prime}\right)\right), \\
J_{A A}^{L} & =\frac{\left(m^{2}-m^{\prime 2}\right)^{2}}{q^{2}}\left(I_{2}-I_{2}^{0}\right)-\left(m+m^{\prime}\right)^{2} I_{2} .
\end{aligned}
$$

For vector mesons, the loop integral is healed by subtracting a certain term $J_{V V}^{T} \rightarrow J_{V V}^{T}-J_{V V}^{(T)}(q=0)+J_{V V}^{(L)}(q=0)$ and one can obtain,

$$
J_{V V}^{T}=\frac{1}{3}\left[2\left(m^{2}+m^{\prime 2}\right)\left(I_{2}-I_{2}^{0}\right)-\left[3\left(m-m^{\prime}\right)^{2}-2 q^{2}\right] I_{2}-\frac{\left(m^{2}-m^{\prime 2}\right)^{2}}{q^{2}}\left(I_{2}-I_{2}^{0}\right)+4\left(m^{2}-m^{\prime 2}\right)^{2} I_{2}^{0^{\prime}}\right] .
$$

The subtracted term tends to zero when one quark mass tends to infinity. The integrations involved are,

$$
\begin{aligned}
& I_{1}(m)=8 i N_{C} \int \frac{\mathrm{d}^{4} p}{(2 \pi)^{4}} \frac{1}{\left(p^{2}-m^{2}+i \epsilon\right)} \\
& I_{2}\left(m, m^{\prime}, q^{2}\right)=4 i N_{C} \int \frac{\mathrm{d}^{4} p}{(2 \pi)^{4}} \frac{1}{\left[\left(p+\frac{1}{2} q\right)^{2}-m^{2}+i \epsilon\right]\left[\left(p-\frac{1}{2} q\right)^{2}-m^{\prime 2}+i \epsilon\right]}
\end{aligned}
$$

and define, $I_{2}^{0}\left(m, m^{\prime}\right) \equiv I_{2}\left(m, m^{\prime}, 0\right)$ and $I_{2}^{0^{\prime}}=\mathrm{d} I_{2} /\left.\mathrm{d} q^{2}\right|_{q^{2}=0}$. After a calculation, one can find, when $\left(m^{\prime}-m\right)^{2}<$ $q^{2}<\left(m+m^{\prime}\right)^{2}$

$$
\begin{aligned}
& I_{1}(m)=\frac{N_{C}}{4 \pi^{2}} \int_{4 m^{2}}^{4\left(\Lambda^{2}+m^{2}\right)} \sqrt{1-\frac{4 m^{2}}{\kappa^{2}}} \mathrm{~d} \kappa^{2} \\
& I_{2}\left(m, m^{\prime}, q^{2}\right)=-\frac{N_{C}}{4 \pi^{2}} \int_{\left(m+m^{\prime}\right)^{2}}^{\left(\sqrt{\Lambda^{2}+m^{2}}+\sqrt{\Lambda^{2}+m^{\prime 2}}\right)^{2}} \frac{\sqrt{1-2 \frac{m^{2}+m^{\prime 2}}{\kappa^{2}}+\left(\frac{m^{2}-m^{\prime 2}}{\kappa^{2}}\right)^{2}}}{\kappa^{2}-q^{2}} \mathrm{~d} \kappa^{2}
\end{aligned}
$$

in which the $\Lambda^{2}$ is the 3 dimensional cutoff. The same expression can be applied to the case $q^{2}<\left(m-m^{\prime}\right)^{2}$.

The integration involved in $0^{-}, 1^{-}$sector is,

$$
j\left(l_{0}\right)=4 i N_{C} \int \frac{\mathrm{d}^{4} k}{(2 \pi)^{4}} \frac{k \cdot v-m_{q}}{\left(k^{2}-m_{q}^{2}+i \epsilon\right)\left(v \cdot k+l_{0}+i \epsilon\right)}
$$

Assuming $v=(1, \overrightarrow{0})$, and integrating out $k_{0}$ below the threshold $l_{0}<m_{q}$, one can find,

$$
j\left(l_{0}\right)=\frac{4 N_{C}}{(2 \pi)^{4}} \pi \int \mathrm{d}^{3} k \frac{\sqrt{\mathbf{k}^{2}+m^{2}}+m_{q}}{\sqrt{\mathbf{k}^{2}+m_{q}^{2}}\left(\sqrt{\mathbf{k}^{2}+m_{q}^{2}}-l_{0}\right)}
$$


Introducing the 3D cutoff, one can get,

$$
j\left(l_{0}\right)=\frac{N_{C}}{(2 \pi)^{2}} \int_{4 m_{q}^{2}}^{4\left(\Lambda^{2}+m_{q}^{2}\right)} \frac{\kappa+2 m_{q}}{2 \kappa-4 l_{0}} \sqrt{1-\frac{4 m^{2}}{\kappa}} \mathrm{d} \kappa^{2}
$$

In which,

$$
\kappa^{2}=4 \mathbf{k}^{2}+4 m_{q}^{2}
$$

[1] N. A. Tornqvist, Phys. Lett. B590, 209 (2004), hep-ph/0402237.

[2] E. S. Swanson, Phys. Lett. B588, 189 (2004), hep-ph/0311229.

[3] X. Liu, Z.-G. Luo, Y.-R. Liu, and S.-L. Zhu, Eur. Phys. J. C61, 411 (2009), 0808.0073.

[4] Y.-R. Liu and Z.-Y. Zhang, Phys. Rev. C79, 035206 (2009), 0805.1616.

[5] S.-H. Yu, B.-K. Wang, X.-L. Chen, and W.-Z. Deng, Chin. Phys. C36, 25 (2012), 1104.4535.

[6] Y. Nambu and G. Jona-Lasinio, Phys. Rev. 122, 345 (1961).

[7] Y. Nambu and G. Jona-Lasinio, Phys. Rev. 124, 246 (1961).

[8] S. P. Klevansky, Rev. Mod. Phys. 64, 649 (1992).

[9] T. Hatsuda and T. Kunihiro, Phys. Rept. 247, 221 (1994), hep-ph/9401310.

[10] U. Vogl and W. Weise, Prog. Part. Nucl. Phys. 27, 195 (1991).

[11] V. Bernard and U. G. Meissner, Nucl. Phys. A489, 647 (1988).

[12] A. H. Blin, B. Hiller, and J. da Providencia, Phys. Lett. B241, 1 (1990).

[13] M. Takizawa, K. Kubodera, and F. Myhrer, Phys. Lett. B261, 221 (1991).

[14] V. Bernard, R. L. Jaffe, and U. G. Meissner, Nucl. Phys. B308, 753 (1988).

[15] S. Klimt, M. F. M. Lutz, U. Vogl, and W. Weise, Nucl. Phys. A516, 429 (1990).

[16] T. Eguchi and H. Sugawara, Phys. Rev. D10, 4257 (1974).

[17] D. Ebert and M. K. Volkov, Z. Phys. C16, 205 (1983).

[18] D. Ebert and H. Reinhardt, Nucl. Phys. B271, 188 (1986).

[19] H. Reinhardt and R. Alkofer, Phys. Lett. B207, 482 (1988).

[20] J. Bijnens, Phys. Rept. 265, 369 (1996), hep-ph/9502335.

[21] N. Isgur and M. B. Wise, Phys. Lett. B232, 113 (1989).

[22] A. Manohar and M. Wise, Heavy Quark Physics (Cambrige University Press, 2000).

[23] M. Neubert, Phys. Rept. 245, 259 (1994), hep-ph/9306320.

[24] D. Ebert, T. Feldmann, R. Friedrich, and H. Reinhardt, Nucl. Phys. B434, 619 (1995), hep-ph/9406220.

[25] A. L. Mota and E. R. Arriola, Eur. Phys. J. A31, 711 (2007), hep-ph/0610146.

[26] M. A. Ivanov, Y. L. Kalinovsky, P. Maris, and C. D. Roberts, Phys. Lett. B416, 29 (1998), nucl-th/9704039.

[27] M. A. Ivanov, Y. L. Kalinovsky, and C. D. Roberts, Phys. Rev. D60, 034018 (1999), nucl-th/9812063.

[28] C. D. Roberts and S. M. Schmidt, Prog. Part. Nucl. Phys. 45, S1 (2000), nucl-th/0005064.

[29] T. Nguyen, N. A. Souchlas, and P. C. Tandy, AIP Conf. Proc. 1116, 327 (2009), 0904.3345.

[30] K. Nakamura et al. (Particle Data Group), J. Phys. G37, 075021 (2010).

[31] E. Gamiz, C. T. H. Davies, G. P. Lepage, J. Shigemitsu, and M. Wingate (HPQCD), Phys. Rev. D80, 014503 (2009), 0902.1815 .

[32] A. Bazavov et al. (Fermilab Lattice and MILC) (2011), 1112.3051.

[33] H. Na et al. (2012), 1202.4914. 